\title{
Control of Vortex Shedding from a Square Cylinder
}

\author{
Chuan Ping Shao* \\ Chinese Academy of Sciences, 100080 Beijing, People's Republic of China \\ and \\ Qing Ding Wei \\ Peking University, 100871 Beijing, People's Republic of China
}

DOI: $\underline{10.2514 / 1.28367}$

\begin{abstract}
Small circular, square, and thin-strip cross-sectional elements are used to suppress vortex shedding from a square cylinder at Reynolds numbers in the range of $1.12 \times 10^{4}-1.02 \times 10^{5}$. The axes of the element and cylinder are parallel. The element's size, position, and angle of attack are varied. Measurements of the fluctuating surface pressures and wake velocities, together with smoke flow visualization, show that vortex shedding from both sides of the cylinder is suppressed and the mean drag and fluctuating lift on the cylinder is reduced if the element is installed in an effective zone downstream of the cylinder. The effective zone of the circular element is shown to be much smaller than those of the other elements. The effects of Reynolds number and blockage ratio are investigated. A phenomenon of monoside vortex shedding is observed. The role of the element's bluffness is investigated and the suppression mechanism is discussed.
\end{abstract}

\begin{tabular}{|c|c|}
\hline & Nomenclature \\
\hline$B$ & side width of the cylinder \\
\hline$b$ & $\begin{array}{l}=\text { width of the strip element, side width of the square } \\
\text { element, or diameter of the circular element }\end{array}$ \\
\hline$C_{D}$ & $\begin{array}{l}\text { mean drag coefficient of the cylinder based on } B \text { as the } \\
\text { reference length }\end{array}$ \\
\hline$C_{d}^{\prime}$ & $\begin{aligned}= & \text { rms value of fluctuating drag coefficient of the cylinder } \\
& \text { based on } B \text { as the reference length }\end{aligned}$ \\
\hline$C_{L}$ & $\begin{array}{l}=\text { mean lift coefficient of the cylinder based on } B \text { as the } \\
\text { reference length }\end{array}$ \\
\hline$C_{l}^{\prime}$ & $\begin{aligned}= & \text { rms value of fluctuating lift coefficient of the cylinder } \\
& \text { based on } B \text { as the reference length }\end{aligned}$ \\
\hline$C_{P}$ & $\begin{array}{l}=\text { mean pressure coefficient based on } V_{\infty} \text { as the reference } \\
\text { velocity }\end{array}$ \\
\hline$C_{p}^{\prime}$ & $\begin{aligned}= & \text { rms value of fluctuating pressure coefficient based on } \\
& V_{\infty} \text { as the reference velocity }\end{aligned}$ \\
\hline$f$ & frequency of fluctuation of pressure or flow velocity \\
\hline $\operatorname{Re}$ & Reynolds number, $V_{\infty} B / v$ \\
\hline$u^{\prime}$ & streamwise component of fluctuating velocity \\
\hline$V_{\infty}$ & mean velocity of oncoming flow \\
\hline$X, Y$ & streamwise and lateral coordinates \\
\hline$\alpha$ & angle of attack of the element \\
\hline$\beta$ & polar angle of the axis position of the element \\
\hline$\lambda$ & distance between the axes of the cylinder and element \\
\hline$v$ & kinematical viscosity of the air \\
\hline
\end{tabular}

\section{Introduction}

$\mathbf{I}^{\mathrm{N}}$ $\mathrm{N}$ THE past decades, great efforts have been devoted to the control of bluff-body wakes, and a number of control methods have been developed [1,2]. Among them is the method of control through an additional small element, which can be classified into two categories: the first category is placing the element upstream of the bluff body, which has been studied by many authors, and the second category is placing the element downstream of the bluff body, which originates in the work of Strykowski and Sreenivasan [3] ]. In

Received 29 November 2006; accepted for publication 27 July 2007. Copyright $(2007$ by the American Institute of Aeronautics and Astronautics, Inc. All rights reserved. Copies of this paper may be made for personal or internal use, on condition that the copier pay the $\$ 10.00$ per-copy fee to the Copyright Clearance Center, Inc., 222 Rosewood Drive, Danvers, MA 01923; include the code 0001-1452/08 $\$ 10.00$ in correspondence with the CCC.

*Associate Professor, Institute of Mechanics; shaocp2005@ yahoo.com.cn.

${ }^{\dagger}$ Professor, State Key Laboratory for Turbulence and Complex Systems.
Strykowski and Sreenivasan's study, a much smaller circular element was set parallel to the main circular cylinder. Vortex shedding from both sides of the main body can be suppressed if the circular element is set in a certain region, called the effective zone. Unfortunately, their results show that the effective zone shrinks as the Reynolds number increases. Vortex shedding cannot be suppressed if the Reynolds number is greater than the order of $10^{2}$. It is claimed in Strykowski and Sreenivasan that the circular element can also suppress vortex shedding from a bluff plate at low Reynolds numbers, but no detailed information is provided.

In our recent work [4-6], a thin strip, rather than a circular element, was set downstream of the main circular cylinder to improve the suppression. Vortex shedding from a circular cylinder can be suppressed at much higher Reynolds numbers if the strip is applied in a region quite similar to the effective zone shown in Strykowski and Sreenivasan [3].

Two questions arise here: Why does the cross-sectional shape of the element have such an important influence on the suppression? Can the improved element method be used to suppress vortex shedding from bodies of sharp edges at high Reynolds numbers?

To answer these questions, small circular, square, and thin-strip cross-sectional elements will be used, and their effects on a square cylinder wake will be compared in the present paper.

\section{Experimental Setup}

Two wind tunnels were used in the experiment: tunnel 1 for the fluctuating surface pressure and wake velocity measurements and tunnel 2 for flow visualization. The test section of tunnel 1 was $11.0 \mathrm{~m}$ long, $1.0 \mathrm{~m}$ high, and $1.2 \mathrm{~m}$ wide. It can supply a uniform freestream of speeds $1.0-30 \mathrm{~m} / \mathrm{s}$ with turbulence intensity of 1.0 $1.2 \%$. A square cylinder of $1.0-\mathrm{m}$ length and side width $B$ was placed at the center of tunnel 1 and spanned the height of the test section. Three square cylinders of $B=40,80$, and $120 \mathrm{~mm}$ were tested. Three kinds of elements of 1.0-m length were used: that is, circular cross-sectional elements of diameter $b=19$ and $14.5 \mathrm{~mm}$; square cross-sectional elements of side widths $b=10,12.5,14.5$, and $19 \mathrm{~mm}$; and thin-strip cross-sectional elements of widths $b=9.6,19$, and $29 \mathrm{~mm}$ and thicknesses of $0.5,1.0$, and $4 \mathrm{~mm}$. The cylinder was made out of Plexiglas square tube of 4-mm thickness. The square, circular, and strip elements were made out of dried pine-tree wood, stainless steel tube, and aluminum plates, respectively. The front surface of the cylinder was at right angles to the oncoming stream. The axes of the element and cylinder were parallel. As shown in Fig. 1, the axis position of the element was determined by Cartesian coordinates $X$ and $Y$ or polar coordinates $\lambda$ and $\beta$. 


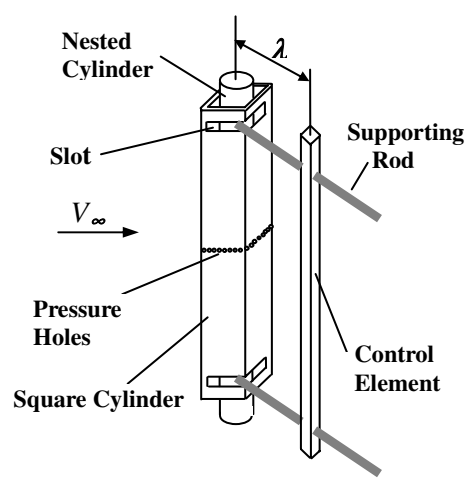

a) Square cylinder and control element

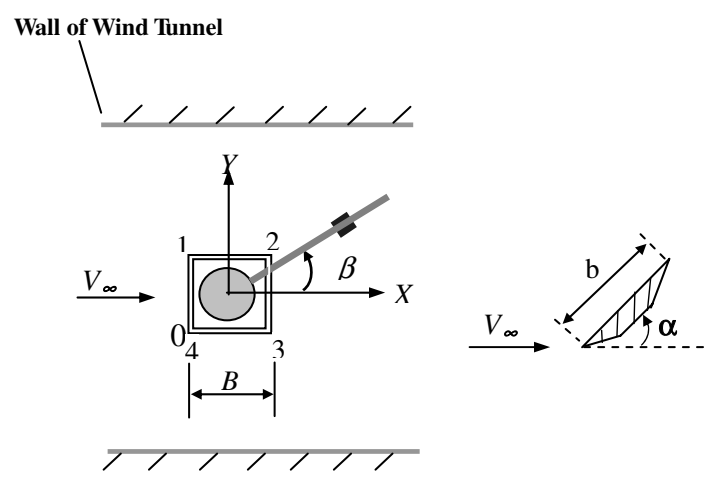

b) Coordinate system c) Strip of trapezoidal
cross section

Fig. 1 Sketch of the square cylinder, control element, and coordinate system.

Two groups of tests were conducted. In the first group, the angle of attack of the square or strip element $\alpha$ is a dependent variable, which changes with the change of polar angle $\beta$ with relation $\alpha=\beta$. In the second group, both the angle of attack $\alpha$ and the axis position of the element are independent variables.

To fix the element in the first group, a circular cylinder of 65.0-mm diameter was nested symmetrically inside the square cylinder. As shown in Fig. 1, steel rods of 5-mm diameter were distributed on a meridian and perpendicularly connected to the surface of the nested circular cylinder. Two rods were used to support the square or circular element, and the distance between the rods was $960 \mathrm{~mm}$. The rods perpendicularly penetrated the square element at midwidth of a side surface, and they perpendicularly and symmetrically penetrated the circular element. Four rods were used to fix the strip element, and the distance between neighboring rods was $320 \mathrm{~mm}$. The long margins of the strip and the axis of the cylinder were parallel and coplanar. Horizontal slots of 6-mm width were made on the square cylinder to allow every rod to move when turning the nested circular cylinder around its axis to adjust the polar angle $\beta$. The slots were sealed by very thin adhesive tape.

No supporter was used in fixing the element in the test of the second group. The ends of the element were in direct contact with the walls of the tunnel. The strip used for independent change of angle $\alpha$ was of 4-mm thickness. As shown in Fig. 1c, the cross section of the strip was trapezoidal, the two sloping sides were of the same length, and the slope angle was $40 \mathrm{deg}$. The longer parallel side of the trapezoidal section was set facing the oncoming flow. The vibrations of the strip and the other elements were negligibly small when the wind speeds were no larger than 5 and $12 \mathrm{~m} / \mathrm{s}$, respectively.

As shown in Fig. 1a, eight pressure holes of 1.2-mm diameter were set on each of the side surfaces of the square cylinder at the section of midspan. The distance between neighboring holes was $9.3 \mathrm{~mm}$ and the distance from the corner of the square cylinder to the nearest hole was $7.5 \mathrm{~mm}$. PVC tubes of $0.8 \mathrm{~mm}$ in inner diameter and $800 \mathrm{~mm}$ in length were set inside the square cylinder to connect the pressure holes to a ZOC 33 electronic pressure-scanning module. The pressures were recorded using a Scanivalve electronic scanner system Hyscan 2000. Each tube was fitted with a restrictor $35 \mathrm{~cm}$ from the pressure hole to improve its high-frequency response. Calibration was carried out before each test. The scanning time of one rotation from the first pressure hole to the last one was $1.6 \mathrm{~ms}$. The sampling frequency and sampling time at each hole were $312 \mathrm{~Hz}$ and $48 \mathrm{~s}$, respectively.

The streamwise component of fluctuating velocity in the wake was measured by a 55P11 sensor of a DANTEC Streamline hot-wire anemometer. The flow unit $90 \mathrm{H} 02$ and calibration module $90 \mathrm{H} 01$ were used to determine the voltage-velocity relation. The temperature compensation system was applied during the measurement, and the uncertainty of the measured velocity was less than $0.5 \%$. Measurements were carried out at downstream stations $X / B=5.0,10.0$, and 15.0, respectively, on the plane of midspan. Points at each station were measured from $Y / B=-2.62$ to 2.62 at an interval of $\Delta(Y / B)=0.435$. The sampling frequency and sampling time were $400 \mathrm{~Hz}$ and $60 \mathrm{~s}$, respectively. Test results show that the

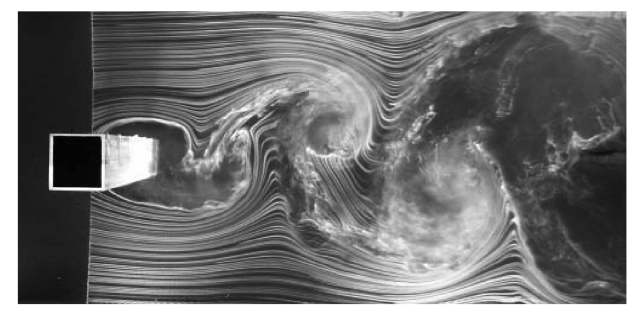

a) Without elements

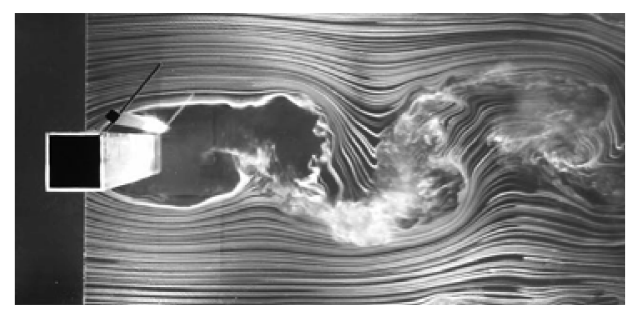

b) $\lambda / B=0.9$

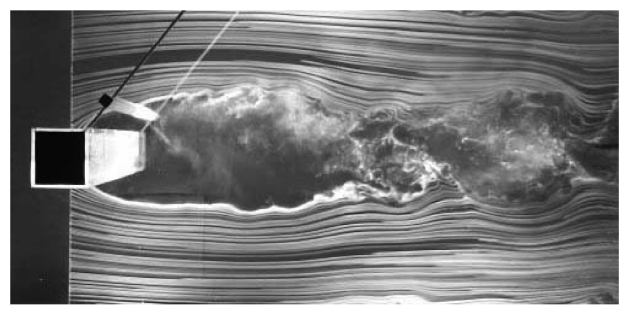

c) $\lambda / B=1.14$

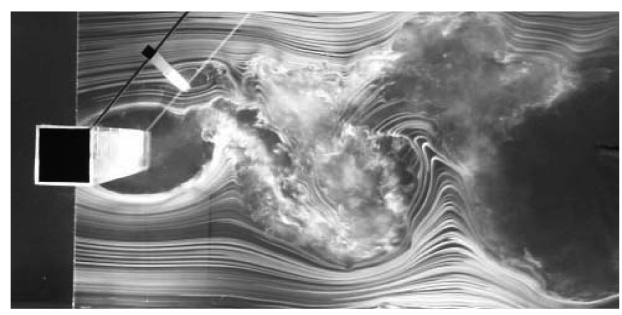

d) $\lambda / B=2.11$

Fig. 2 Suppression effects of a square element at different $\lambda / B$ distances. $b / B=0.18, \alpha=\beta=50 \mathrm{deg}$, and $R e=1.12 \times 10^{4}$, and the blockage ratio is $11.8 \%$. 
Table 1 Uncertainties of pressures measured by tube system at different oncoming velocities

\begin{tabular}{llcc}
\hline \hline Oncoming velocity $V_{\infty}$ & $5.0 \mathrm{~m} / \mathrm{s}$ & $10.0 \mathrm{~m} / \mathrm{s}$ & $15.0 \mathrm{~m} / \mathrm{s}$ \\
$\begin{array}{l}\text { Uncertainty of mean pressure } \\
\text { coefficient }\end{array}$ & $1.1 \%$ & $0.9 \%$ & $0.8 \%$ \\
$\begin{array}{c}\text { Uncertainty of fluctuating } \\
\text { pressure coefficient }\end{array}$ & $2.4 \%$ & $1.8 \%$ & $1.4 \%$ \\
\hline \hline
\end{tabular}

power spectra of fluctuating velocities at any of the stations can provide sufficient information to indicate whether vortex shedding exists in the wake. The results of measurements at station $X / B=$ 10.0 were used in the following discussion.

Smoke-wire visualization was carried out in tunnel 2 . The test section was $5 \mathrm{~m}$ long, $0.6 \mathrm{~m}$ high, and $0.6 \mathrm{~m}$ wide. The test section could supply a uniform freestream of speeds $1.0-25.0 \mathrm{~m} / \mathrm{s}$, with turbulence intensity less than $0.2 \%$. The side width of the cylinder was $60 \mathrm{~mm}$. The installations of the cylinder and control elements were similar to those in tunnel 1 , but six supporting rods, with a neighboring distance of $115 \mathrm{~mm}$, were used in fixing a strip. A single smoke wire was set in front of the cylinder, and the flow at the midspan was visualized.

The uncertainty of the pressure measured by the PVC tube system was investigated in freestream without a cylinder model. The orifice at one end of each tube was directly facing the oncoming stream, and the other end was connected to the ZOC 33 module. By comparing the mean and fluctuating pressures measured by the tube system and the mean and fluctuating pressures calculated from the mean and fluctuating velocities of the oncoming stream measured by the hotwire anemometer, we can evaluate the uncertainties. The uncertainties of mean and fluctuating pressures at different velocities of stream are shown in Table 1. The differences among the lift (or drag) coefficients calculated by Simpson, trapezoidal, and Fourier integrations of the pressure distribution around the cylinder are less than $2.1 \%$.

So the uncertainties of mean and fluctuating lift (drag) coefficient are less than 3.2 and $4.5 \%$, respectively. The uncertainty of Reynolds number, caused by the variations of temperature of the wind air during the tests, was less than $2 \%$.

\section{Results}

The influence of supporting rods on the flow at the section of midspan was shown to be negligibly small. The variable parameters are element size $b / B$, angle of attack $\alpha$, and element position characterized by $X / B$ and $Y / B$ or $\lambda / B$ and $\beta$. The ranges of $\lambda / B$ and $\beta$ tested in the first group were $0.8-2.3$ and $0-90 \mathrm{deg}$, respectively, and the ranges of $X / B$ and $Y / B$ tested in the second group were $0-2.5$ and $0-2.5$, respectively. The test Reynolds number range is $1.12 \times 10^{4}-1.02 \times 10^{5}$.

\section{A. Effect of the Square Element}

Figure 2 shows the effect of distance $\lambda / B$ at $\alpha=\beta=50 \mathrm{deg}$, $b / B=0.18$, and $R e=1.12 \times 10^{4}$. Vortex shedding naturally occurs behind the cylinder without elements (Fig. 2a). Vortex shedding cannot be suppressed if the square element is placed very close to the cylinder $(\lambda / B<0.98)$, but the vortex formation length [7] increases and the wake width decreases as $\lambda / B$ increases. Transition from vortex-shedding wake to suppressed wake happens at $\lambda / B=0.98-1.06$. Large-scale vortex shedding from both sides is suppressed and the wake is narrowed in the range of $1.06<\lambda / B<1.63$. A phenomenon of monoside vortex shedding occurs at $\lambda / B=1.63-2.11$. The shear layer on the upper side is diffused and vortex shedding on the upper side is suppressed. However, large-scale vortex shedding is still present on the lower side.

Figure 3 examines the effect of angle $\alpha=\beta$ at $b / B=0.18$, $\lambda / B=1 . \overline{56}$, and $\operatorname{Re}=3.36 \times 10^{4}$. At angles $\alpha=\beta \leq 35 \mathrm{deg}$, the element is well inside the cylinder wake, and vortex shedding is not suppressed. At $\alpha=\beta=40 \mathrm{deg}$, vortex shedding is still not suppressed, but the shear layer on the upper side is diffused as it curves inward in generating vortex roll-up. Vortex shedding from both sides is suppressed in the range $\alpha=\beta=43-55$ deg. However, irregular vortices or waves still appear, due to the instability of the separated shear layers [8]. The element is covered up by the cylinder wake at $\alpha=\beta=43-45 \mathrm{deg}$, but it is outside the wake at $45 \operatorname{deg}<\alpha=\beta<55 \mathrm{deg}$. Monoside vortex shedding occurs in the range $55<\alpha=\beta \leq 90 \mathrm{deg}$. A small-scale vortex street appears behind the element when $60 \leq \alpha=\beta \leq 90 \mathrm{deg}$, but it smears and then disappears after a short distance.

Figure 4 shows the effect of the size ratio of the square element at $\lambda / B=1 . \overline{5} 6$ and $\alpha=\beta=55 \mathrm{deg}$. Although the shear layer on the upper side is diffused by both the elements, the suppression effect is quite different. The larger $b / B$ can suppress vortex shedding from both sides; however, the smaller $b / B$ cannot suppress vortex shedding from either side.

From Figs. 2 and 3 we may deduce that an effective zone of the square-element position exists in which vortex shedding from both sides of the cylinder is suppressed. Hot-wire measurements for all element positions were performed at various points of $Y / B$ in the wake. In the first group, the tested element positions form a net with intervals $\Delta \beta=2.5-5 \mathrm{deg}$ and $\Delta(\lambda / B)=0.1-0.2$ that covers the

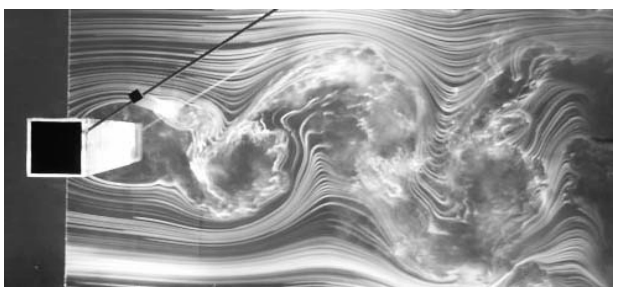

a) $\alpha=\beta=40 \mathrm{deg}$

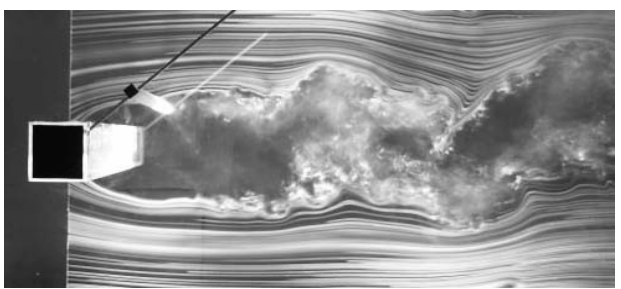

b) $\alpha=\beta=43 \mathrm{deg}$

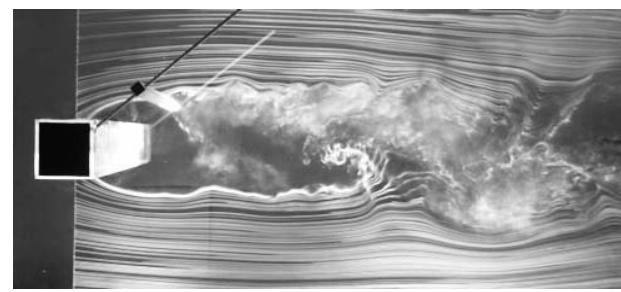

c) $\alpha=\beta=45 \mathrm{deg}$

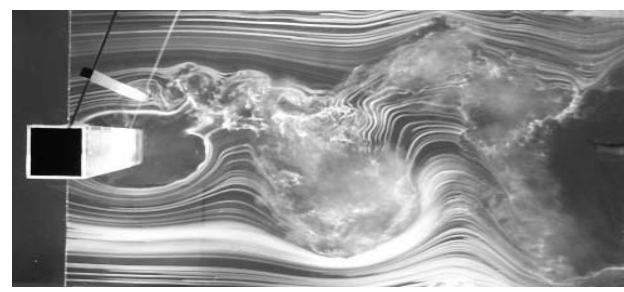

d) $\alpha=\beta=70 \mathrm{deg}$

Fig. 3 Suppression effects of a square element at different angles of $\alpha=\beta, b / B=0.18, \lambda / B=1.56$, and $R e=3.36 \times 10^{4}$, and the blockage ratio is $11.8 \%$. 


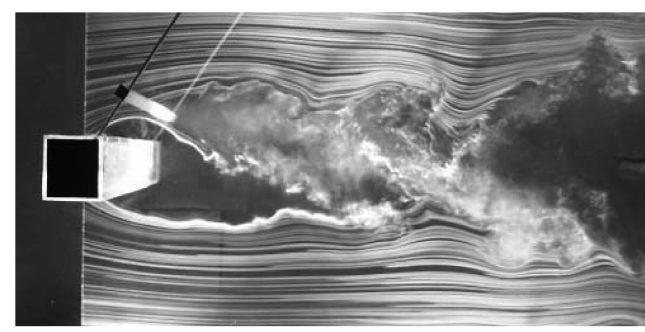

a) $b / B=0.18$

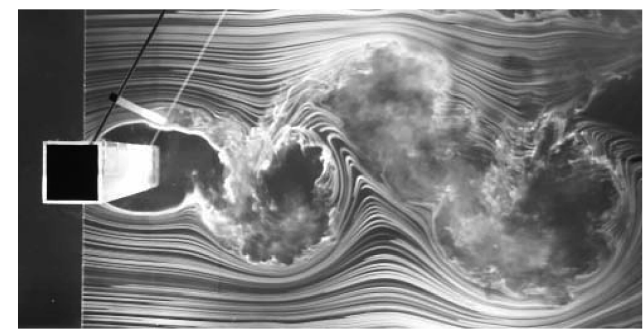

b) $b / B=0.125$

Fig. 4 Comparison of suppression effects between square elements of $b / B=0.125$ and $b / B=0.18$ at $\lambda / B=1.56, \alpha=\beta=55$ deg, and $R e=1.12 \times 10^{4}$, and the blockage ratio is $11.8 \%$.

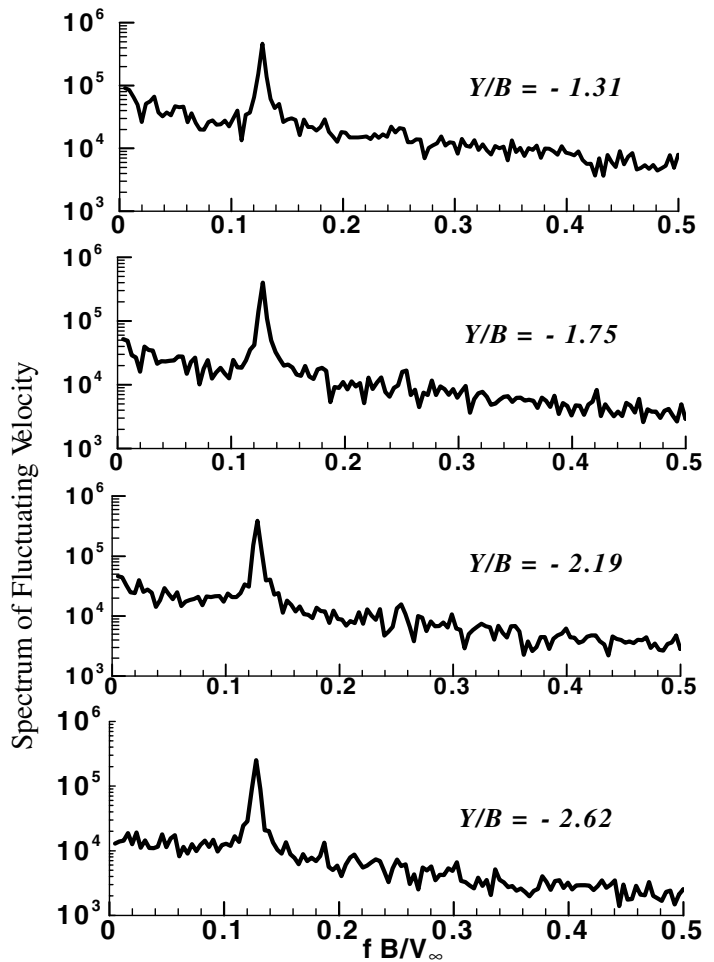

a) Without element

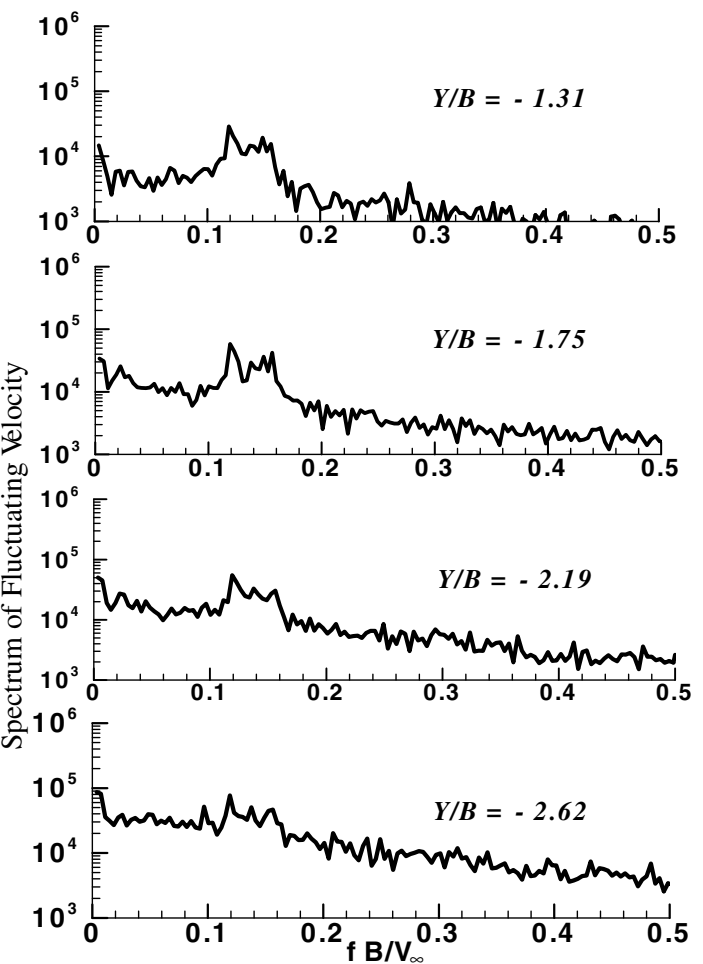

b) With a square element of $b / B=0.18$ at $\lambda / B=1.56$ and $\alpha=\beta=50 \mathrm{deg}$

Fig. 5 Power spectra of fluctuating velocities $\left(u^{\prime} / V_{\infty}\right)$ at different $Y / B$ points in wakes without an element and with a square element of $b / B=0.18$ at $\lambda / B=1.56 ; \alpha=\beta=50 \mathrm{deg}$, measured at station $X / B=10.0$ on the plane of midspan; $R e=3.36 \times 10^{4}$; and the blockage ratio is $11.8 \%$.

area of $0 \mathrm{deg} \leq \beta \leq 90 \mathrm{deg}$ and $0.8 \leq \lambda / B \leq 2.3$. In the second group, the net has intervals of $\Delta(X / B)=0.06$ and $\Delta(Y / B)=0.03$ that cover the area of $0 \leq X / B \leq 2.5$ and $0 \leq Y / B \leq 2.5$. As shown in Fig. 5a, a sharp peak appears in each of the power spectra of fluctuating velocities at $Y / B=-1.31--2.6$ when no element is applied. The frequency at the peak is $f B / V_{\infty} \approx 0.13$, close to the Strouhal number measured by other authors $[9,10]$. On the other hand, when vortex shedding is suppressed, the velocity fluctuations at all points are weakened and no sharp peaks appear in the power spectra (Fig. 5b). If we combine this criterion with the results of visualization, we can determine the effective zone. Effective zones of the square elements of different $b / B$ widths are shown in Fig. 6 . The effective zone is very small for $b / B=0.125$, but it enlarges rapidly with the increase of $b / B$ from 0.125 to 0.24 .

The phenomenon of monoside vortex shedding is confirmed by hot-wire measurements. The power spectra of fluctuating velocities in a typical wake are demonstrated in Fig. 7. No sharp peaks appear in the spectra at points on the upper side $(Y / B>0)$ of the wake. On the lower side $(Y / B<0)$, however, a sharp peak appears in each of the spectra measured at $Y / B=-1.31--2.62$. The peak frequency is $f B / V_{\infty} \approx 0.13$, nearly the same as that without elements.

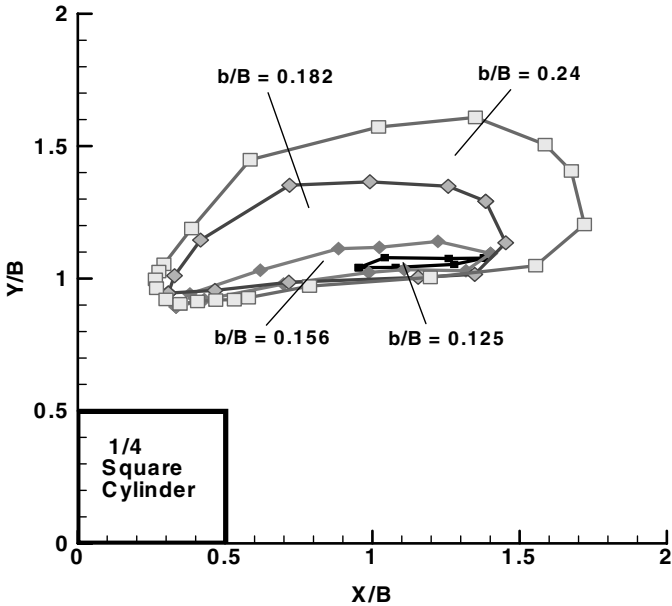

Fig. 6 Effective zones of square elements of different $b / B$ widths, $R e=3.36 \times 10^{4}, \alpha=\beta$ at every position, and the blockage ratio is $11.8 \%$. 

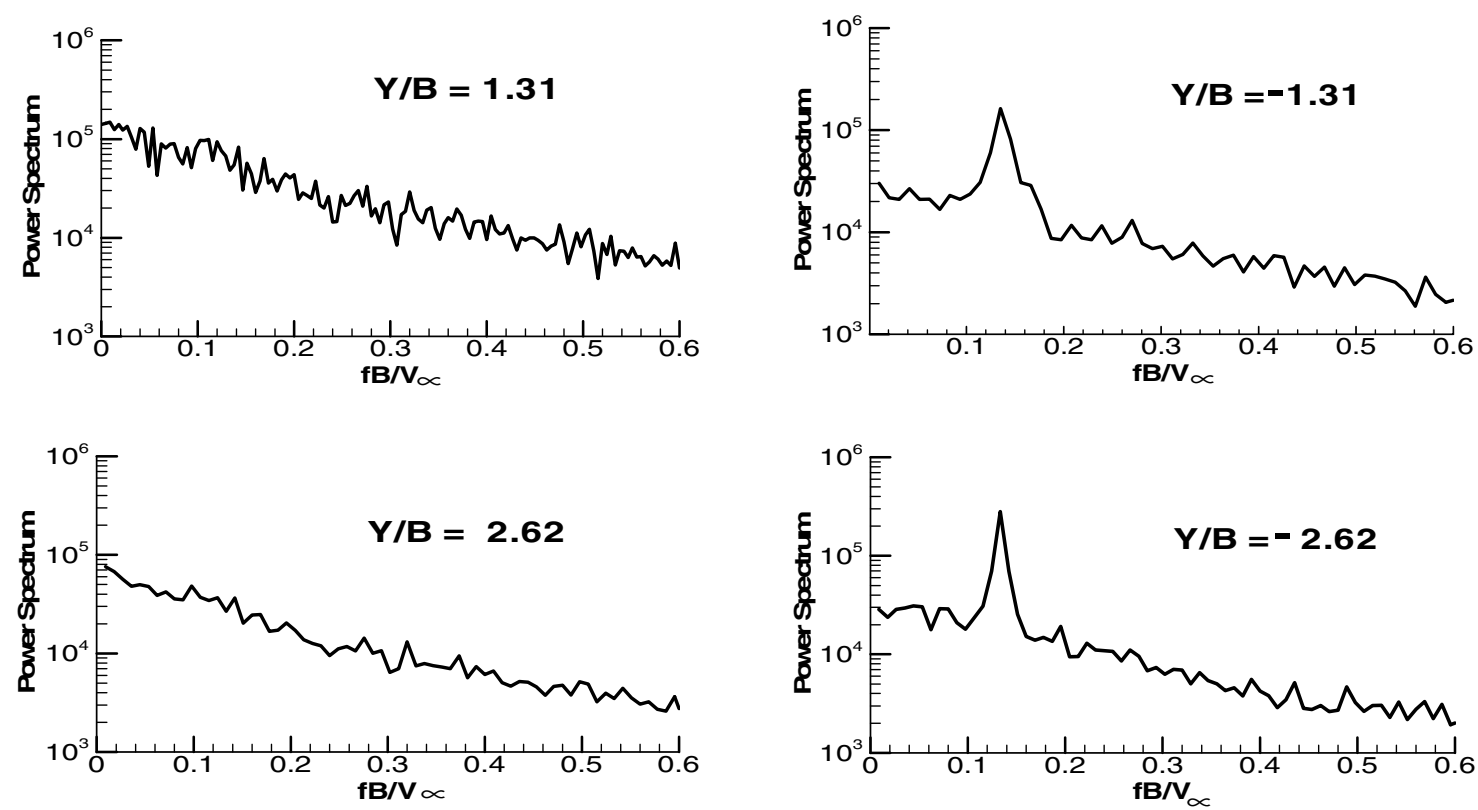

Fig. 7 Power spectra of fluctuating velocities $\left(u^{\prime} / V_{\infty}\right)$ at different $Y / B$ points on the upper $(Y / B>0)$ and lower $(Y / B<0)$ sides of the wake controlled by a square element of $b / B=0.18$ at $\lambda / B=1.62 ; \alpha=\beta=65 \mathrm{deg}$, measured at station $X / B=10.0$ on the plane of midspan; Re $=3.36 \times 10^{4}$; and the blockage ratio is $4.13 \%$.

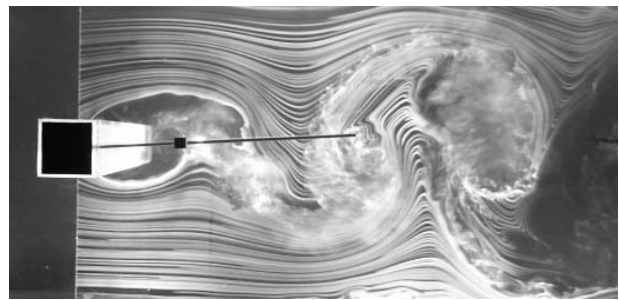

a) $\lambda / B=1.70$ and $\beta=0 \mathrm{deg}$

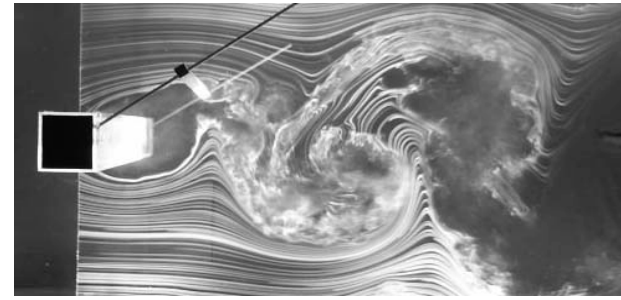

b) $\lambda / B=\mathbf{2 . 0 5}$ and $\beta=\mathbf{4 0 ~ d e g}$

Fig. 8 Effect of a square element of $b / B=0.18$ at angles $\alpha=\beta \leq 40 \mathrm{deg}, R e=1.12 \times 10^{4}$, and the blockage ratio is $11.8 \%$.

The effect of a splitter plate set on the centerline of the wake is said to separate the shear layers and prevent their interactions $[3,8]$. As shown in Fig. 8, the square element has little function in blocking the way of vortex roll-up and preventing interactions between the shear layers when it is at angles of $\alpha=\beta$ not larger than $40 \mathrm{deg}$.

\section{B. Comparisons with the Other Elements}

As shown in Fig. 9a, vortex shedding is not markedly influenced by the row of supporting rods. However, it is suppressed and the wake is narrowed when a thin-strip element is applied and set in an effective zone downstream of the cylinder. Figure $9 \mathrm{~b}$ is a typical case of suppression at $b / B=0.18, \lambda / B=1.56, \alpha=\beta=45 \mathrm{deg}$, and $R e=1.12 \times 10^{4}$.

As shown in Fig. 10, vortex shedding cannot be suppressed by a circular element of $b \overline{/ B}=0.18$ at any point within the range tested.
The effective zones of the three elements of size ratio $b / B=0.24$ are determined and shown in Fig. 11. Compared with the zones of the other elements, the zone of the circular element is much smaller, with its main part enclosed in the zone of the square element.

The "bluffness" of the element may play a role in the suppression. In Roshko's [11] definition, when two bodies have the same frontal area, the bluffer one tends to diverge the flow more, create a broader wake, experience a higher drag, and have a lower value of Strouhal number. Table 2 lists the values of drag coefficient $C_{D b}$ and Strouhal number of the three elements at different angles $\alpha$ over Reynolds number $R e_{b}$ range of $2.6 \times 10^{3}-8.0 \times 10^{3}$, where $R e_{b}, C_{D b}$, and $S t_{b}$ are based on $V_{\infty}$ and size $b$ of the elements. Of the three elements of the same $b / B$, the circular element experiences the lowest drag and has the highest value of Strouhal number in the range of $\alpha=\beta=40-90 \mathrm{deg}$. The low bluffness of the circular element may be the reason for its poor suppression effect. Of the four square

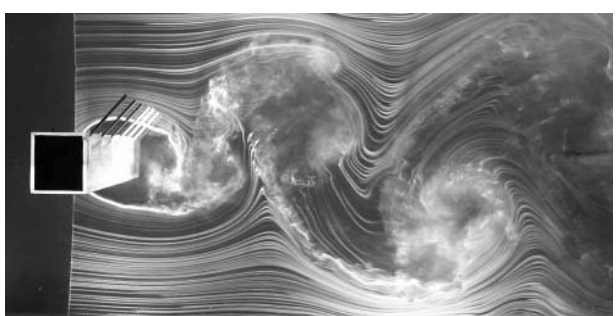

a) Cylinder with solely rods, $\alpha=\beta=45 \mathrm{deg}$

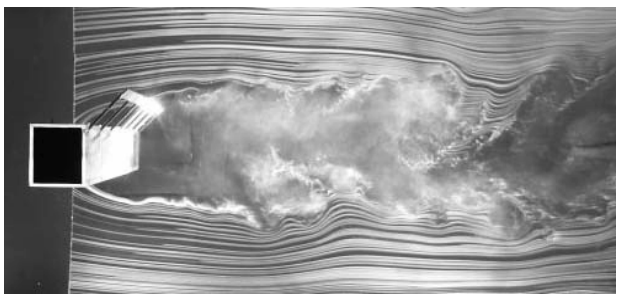

b) Cylinder with a strip element of $b / B=0.18$, placed at $\lambda B=1.56, \alpha=\beta=45 \mathrm{deg}$

Fig. 9 Effect of a strip element at $R e=1.12 \times 10^{4}$, and the blockage ratio is $11.8 \%$. 


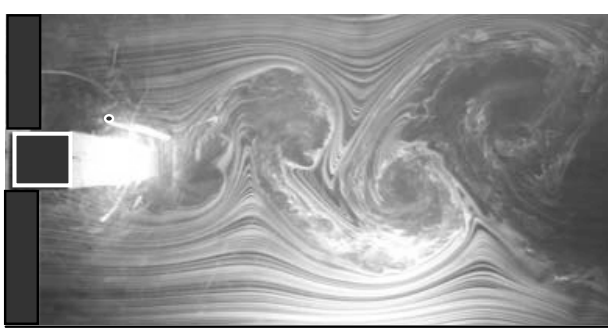

a) $X / B=1.0$ and $Y / B=0.75$

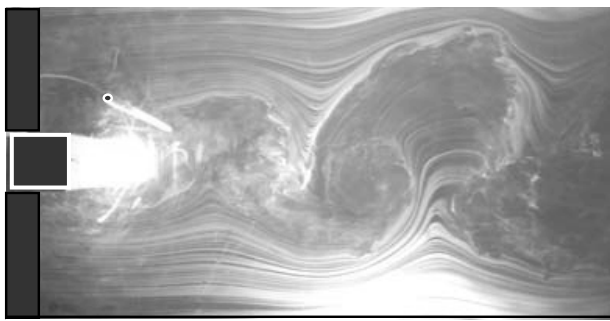

b) $\mathrm{X} / \mathrm{B}=1.0$ and $\mathrm{Y} / \mathrm{B}=1.11$

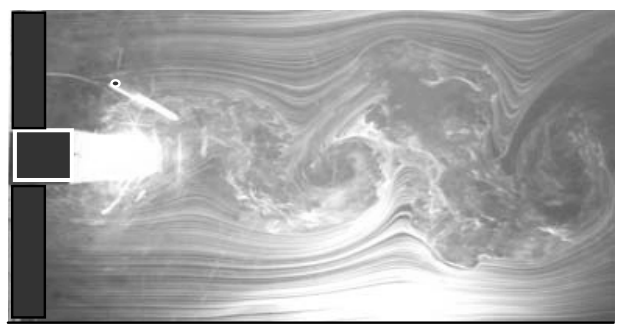

c) $X / B=1.0$ and $Y / B=1.31$

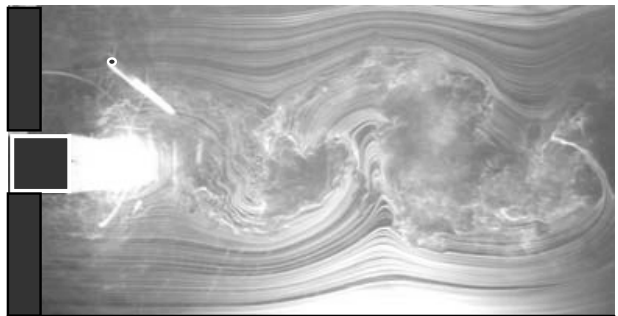

d) $X / B=1.0$ and $Y / B=1.56$

Fig. 10 Effect of a circular element of $b / B=0.18$ at different positions, $R e=1.12 \times 10^{4}$, and the blockage ratio is $4.13 \%$; visualized in tunnel 1 .

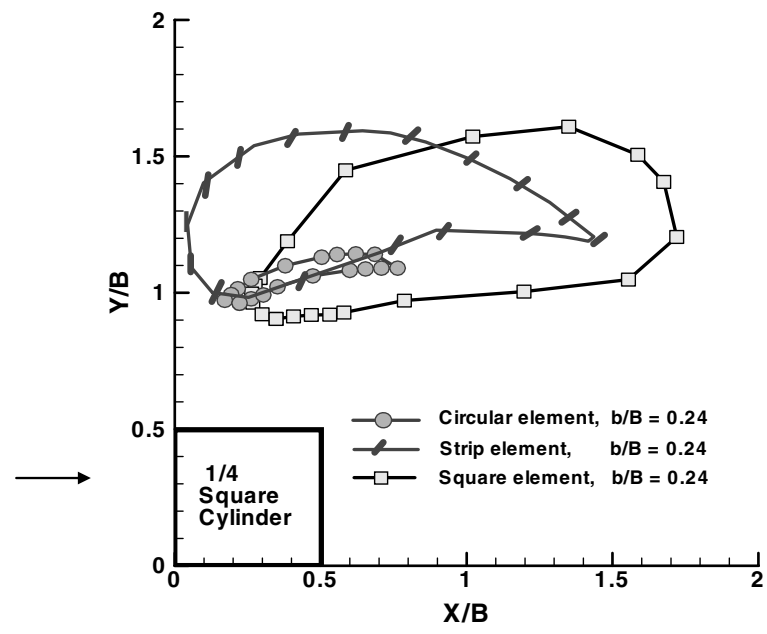

Fig. 11 Effective zones of the three elements of $b / B=0.24$, $R e=3.36 \times 10^{4}, \alpha=\beta$ at every position, and the blockage ratio is $8.27 \%$.

elements, the one with the largest $b / B$ has the greatest bluffness and best suppression effect (Figs. 4 and 6). The main part of the effective zone of the strip element is ahead of that of the square element. This may be attributed to the increase in bluffness of the strip element with the increase of $\beta$ from 0 to $90 \mathrm{deg}$ and the decrease in bluffness of the square element with the increase of $\alpha=\beta$ from 45 to $77 \mathrm{deg}$.

Figure 12 shows the effect of angle $\alpha$ of a strip of trapezoidal cross section. Vortex shedding on both sides of the cylinder cannot be suppressed when $\alpha \leq 30 \mathrm{deg}$. At $\alpha=45 \mathrm{deg}$, vortex shedding on the upper side is suppressed, but it cannot be suppressed on the lower side. At $\alpha=60-9$ deg, large-scale regular vortex shedding on both sides is suppressed, and there appear only small-scale or irregular vortices in the wake.

The effective zone of a strip of trapezoidal cross section at a fixed angle $\alpha$ was also studied at $R e=3.36 \times 10^{4}$. The effective zones at

Table 2 Values of drag and Strouhal number of the elements

\begin{tabular}{lcc}
\hline \hline \multicolumn{1}{c}{ Section of the element } & $C_{D b}$ & $S t_{b}$ \\
\hline Circular & $1.05-1.1$ & $0.21-0.20$ \\
Thin strip $[12,13], \alpha=40-90 \mathrm{deg}$ & $1.2-2.0$ & $0.19-0.13$ \\
Square $\left[\underline{9}, \frac{10}{10}, \alpha=45-77 \mathrm{deg}\right.$ and & $2.2-1.6$ and & $0.13-0.16$ and \\
$\alpha=7 \overline{7}-90 \mathrm{deg}$ & $1.6-2.0$ & $0.16-0.14$ \\
\hline \hline
\end{tabular}

$\alpha=35,45,60$, and $90 \mathrm{deg}$ are shown in Fig. 13 . The zone of $\alpha=90$ deg is slightly broader than the zone of strip shown in Fig. 11 . The zone shrinks rapidly with the decrease of $\alpha$ from 90 to $35 \mathrm{deg}$. It is interesting to note that the zone is split into two small regions when $\alpha$ is reduced below a critical value between 45 and $60 \mathrm{deg}$.

The downstream region of the zone at $\alpha=35-45 \mathrm{deg}$ is enclosed in the zone at larger $\alpha$. However, the upstream region of the zone at $\alpha=35-45 \mathrm{deg}$ is closer to the cylinder than the zone at larger $\alpha$. Test results show that the effective zone does not exist for $\alpha \leq 30 \mathrm{deg}$.

For a square element, the suppression effect also changes with a change of angle $\alpha$, but it is not as sensitive as that of a strip element.

\section{Blockage Ratio Effect}

The influence of the blockage ratio of the model and element on the effective zone of a square element at fixed angle of attack $\alpha=90 \mathrm{deg}$ was studied in tunnel 1 . Three square cylinders of side width $B=40,80$, and $120 \mathrm{~mm}$ were tested, respectively. As shown in Fig. 14, the effective zone moves upstream and inward with the increase of blockage ratio.

\section{Effect of Reynolds Number}

The influence of Reynolds number on the effective zone of a square element of $b / B=0.24$ at $\alpha=90 \mathrm{deg}$ was also studied. The Reynolds number cannot be increased by solely increasing the velocity of wind stream, because a high stream velocity $\left(V_{\infty} \geq 15 \mathrm{~m} / \mathrm{s}\right)$ may induce strong vibrations of the element. Two square cylinder models of width $B=40$ and $120 \mathrm{~mm}$ were tested, and the results are shown in Figs. $15 \mathrm{a}$ and $15 \mathrm{~b}$ respectively. From Fig. 15 we know that the effective zone slightly enlarges with an increase of Reynolds number.

\section{E. Effects on Fluctuating Pressures}

Figure 16a shows the power spectra of fluctuating pressure coefficients measured at different points on the side surfaces of the cylinder without elements. A sharp peak appears in each of the spectra, indicating the presence of vortex shedding. The peak frequency $f B / V_{\infty} \approx 0.13$ is the same as the Strouhal number obtained from measurements of fluctuating velocities in the wake.

Figure 16b shows the power spectra of fluctuating pressure coefficients at different points on the side surfaces of the cylinder with a square element of $b / B=0.24$ at $\lambda / B=1.31$ and $\alpha=\beta=60 \mathrm{deg}$. There are no sharp peaks in any of the spectra. This is a typical case demonstrating that regular vortex shedding is effectively suppressed. 


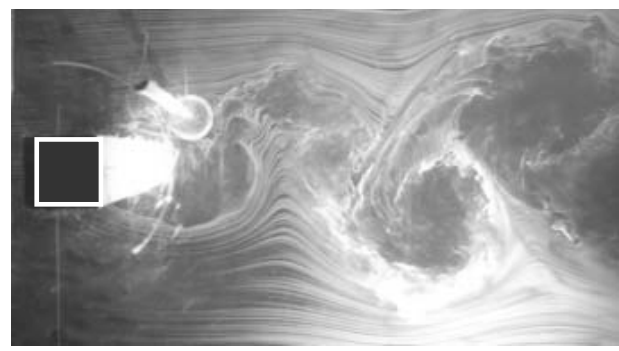

a) $\alpha=30 \mathrm{deg}$

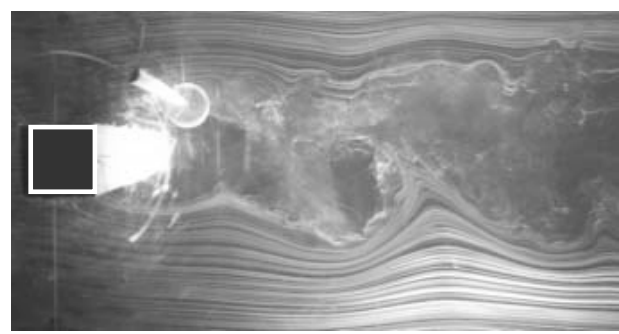

b) $\alpha=45 \mathrm{deg}$

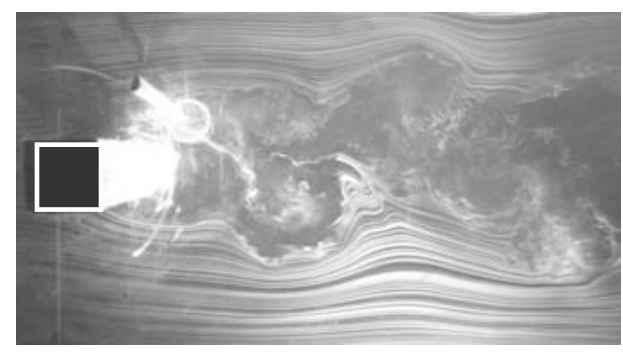

c) $\alpha=60 \mathrm{deg}$

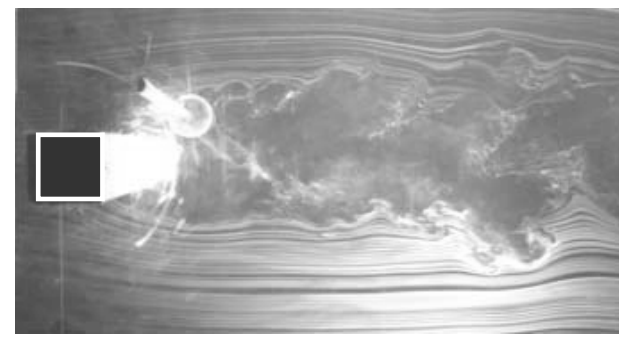

d) $\alpha=60 \mathrm{deg}$

Fig. $12 \alpha$ angle effect of a strip of trapezoidal cross section at a fixed position $X / B=1.0, Y / B=1.25, b / B=0.24$, and $R e=1.12 \times 10^{4}$, and the blockage ratio is $4.13 \%$; visualized in tunnel 1 .

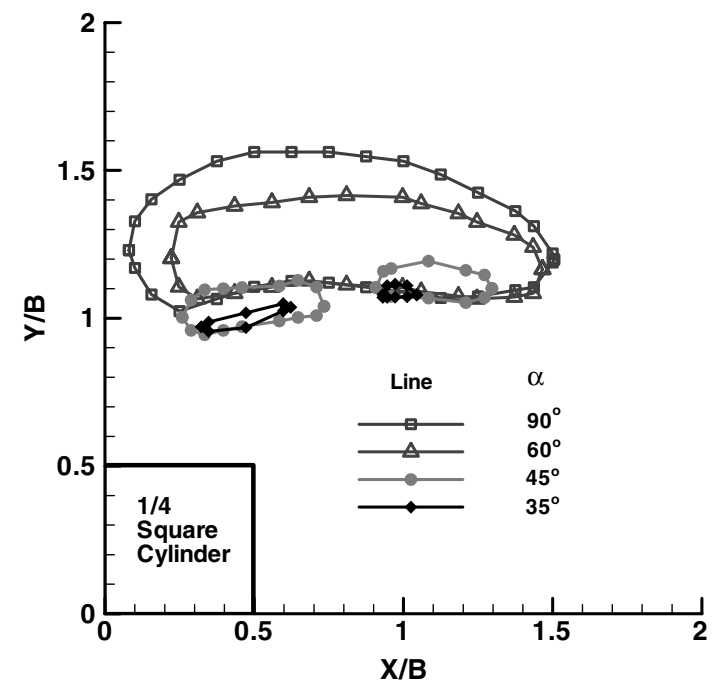

Fig. 13 Effective zones of a strip of trapezoidal cross section at different $\alpha$ angles, $b / B=0.24, R e=3.36 \times 10^{4}$, and the blockage ratio is $8.27 \%$.

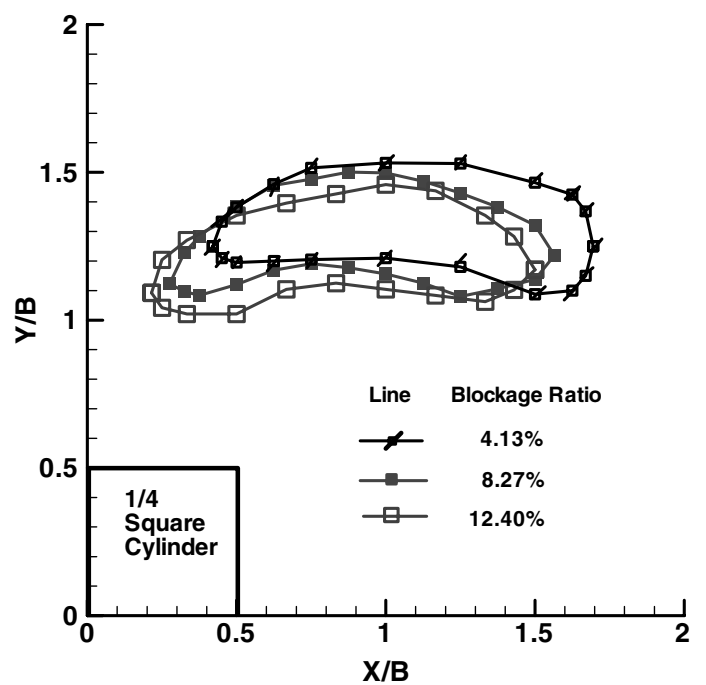

Fig. 14 Influence of blockage ratio on effective zones of a square element at $\alpha=90 \mathrm{deg}, b / B=0.24$, and $R e=3.36 \times 10^{4}$.

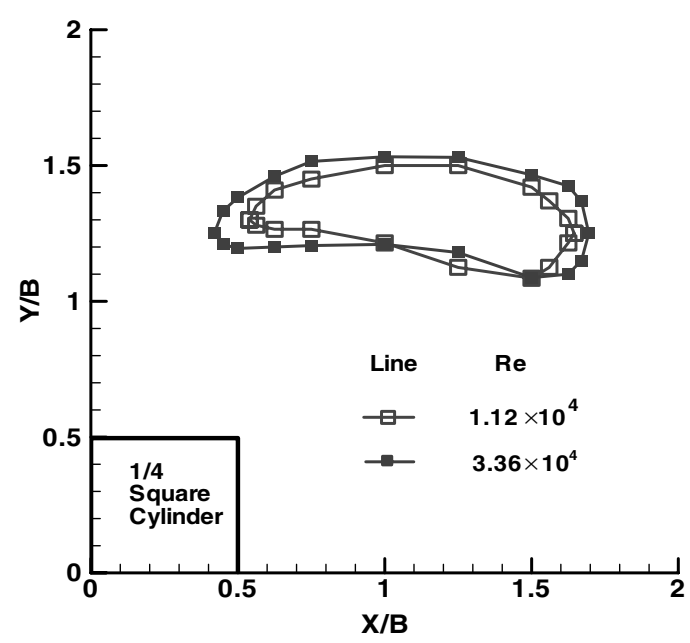

a) Blockage ratio $=\mathbf{4 . 1 3 \%}$

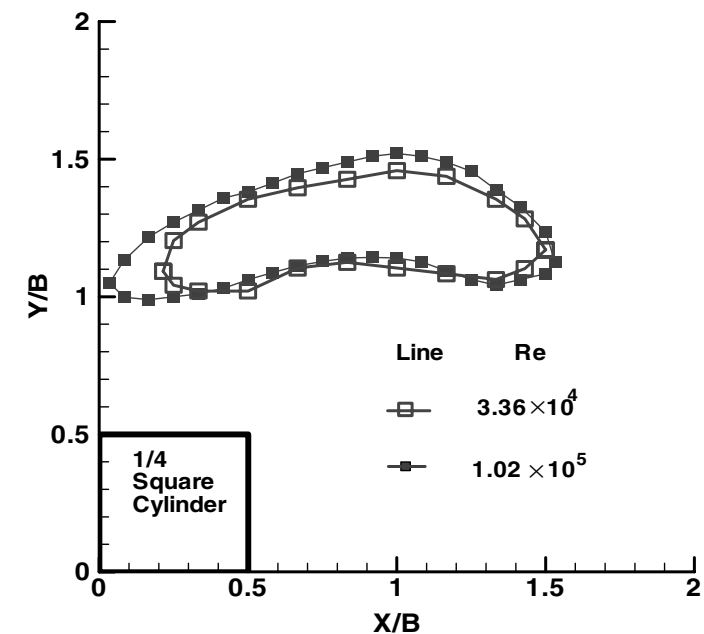

b) Blockage ratio $=\mathbf{1 2 . 4 0 \%}$

Fig. 15 Influence of Reynolds number on the effective zone of the square element of $b / B=0.24$ at $\alpha=90$ deg and different blockage ratios. 


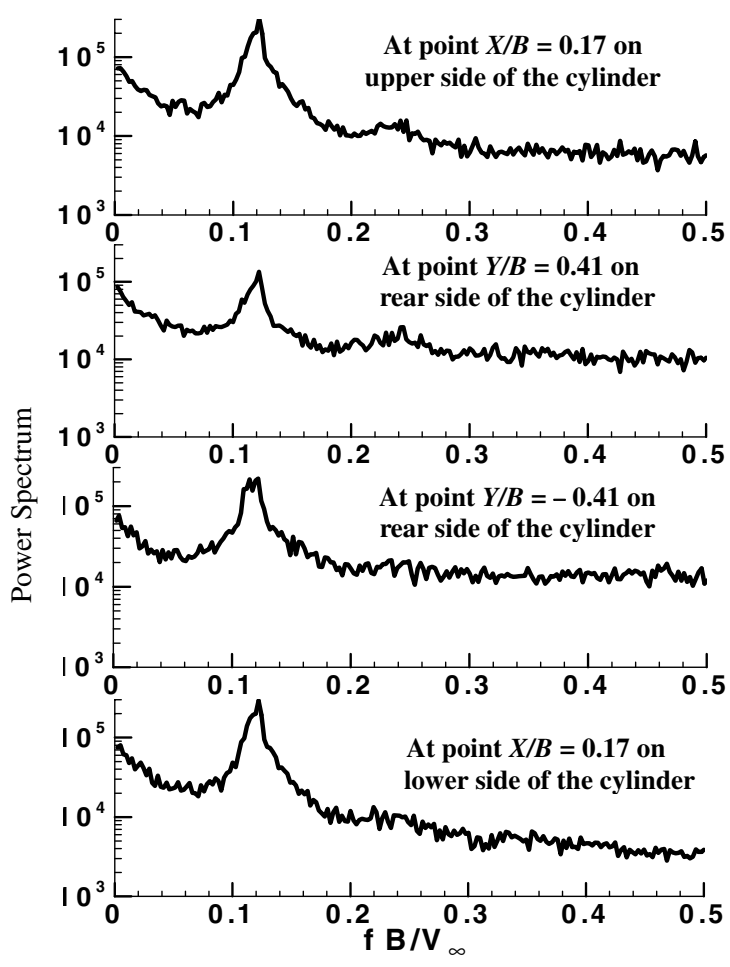

a) Without element

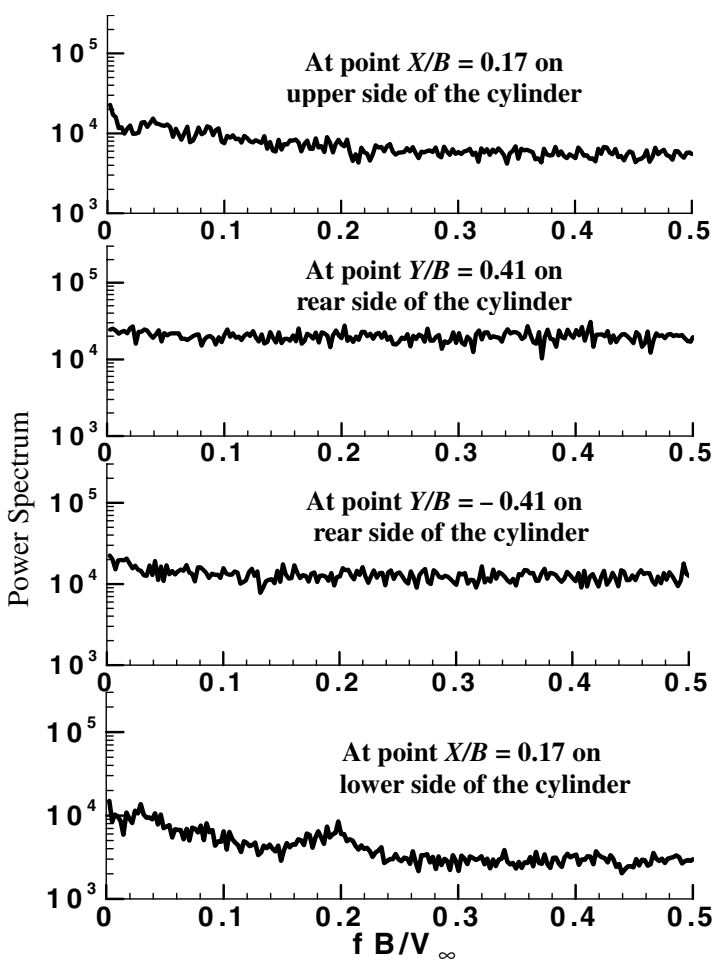

b) With a square element of $b / B=0.24$, placed at $\lambda / B=1.31$ and $\alpha=\beta=60 \mathrm{deg}$

Fig. 16 Power spectra of fluctuating pressures measured at different points on side surfaces of the cylinder without and with a control element, $R e=5.6 \times 10^{4}$, and the blockage ratio is $8.27 \%$.

The angle $\alpha=\beta$ effects of the three elements of $b / B=0.24$ on the distribution of the rms value of fluctuating pressure coefficient $C_{p}^{\prime}$ are shown in Figs. 17a-17c. Compared with the case without elements, $C_{p}^{\prime}$ is greatly reduced by the square element in $40 \mathrm{deg} \leq$ $\alpha=\beta \leq 70 \mathrm{deg}$ and by the strip element in $40 \mathrm{deg} \leq \alpha=$ $\beta<85 \mathrm{deg}$. However, if the circular element is applied, obvious reduction of $C_{p}^{\prime}$ happens only in a narrow range in the vicinity of $\alpha=\beta=50 \mathrm{deg}$.
F. Suppression of the Lift and Drag Fluctuations

The lift and drag on the cylinder are obtained by integrating the surface pressures; Fig. 18 a shows the angle $\alpha=\beta$ effect of a square element of $b / B=0.24$ on the power spectra of fluctuating lift and drag coefficients. A high peak appears in the spectrum of fluctuating lift without the presence of the element or in the spectrum at a small angle of attack of the element. The peak value of the spectrum reduces with the increase of $\alpha=\beta$ from 0 to $40 \mathrm{deg}$. The sharp peak

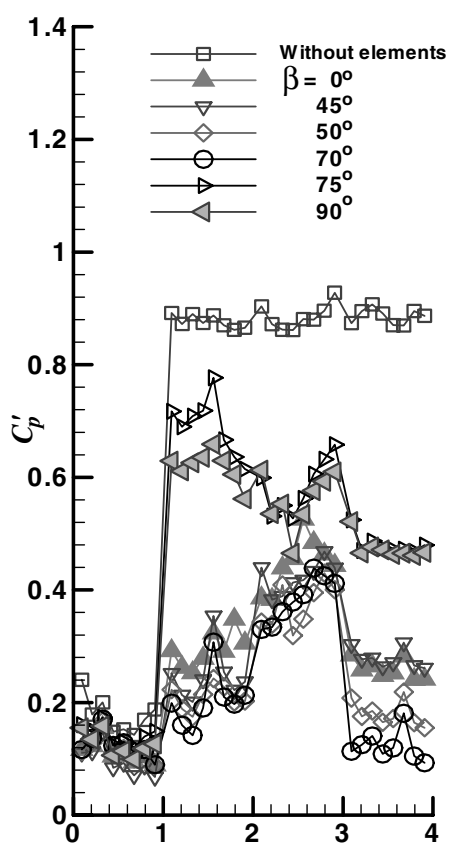

a) With a square element

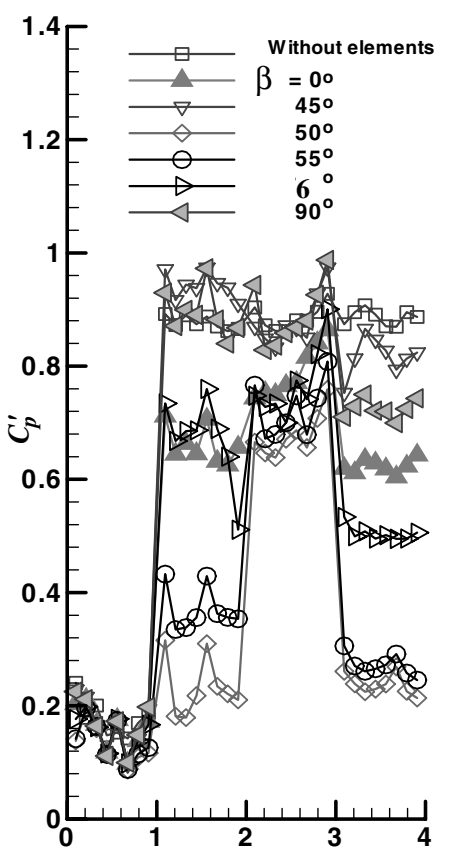

b) With a circular element

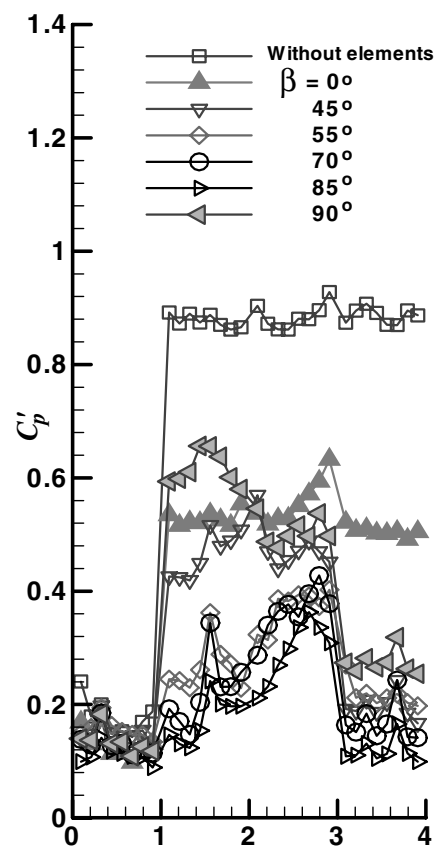

c) With a strip element

Fig. 17 Angle $\alpha=\beta$ effect of the three elements of $b / B=0.24$ on distribution of rms value, and the blockage ratio is $8.27 \%$. 


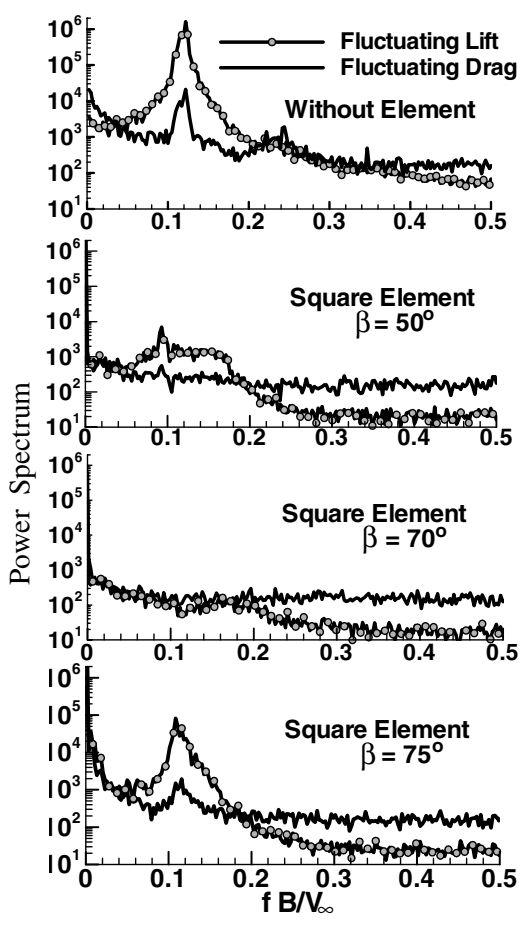

a)
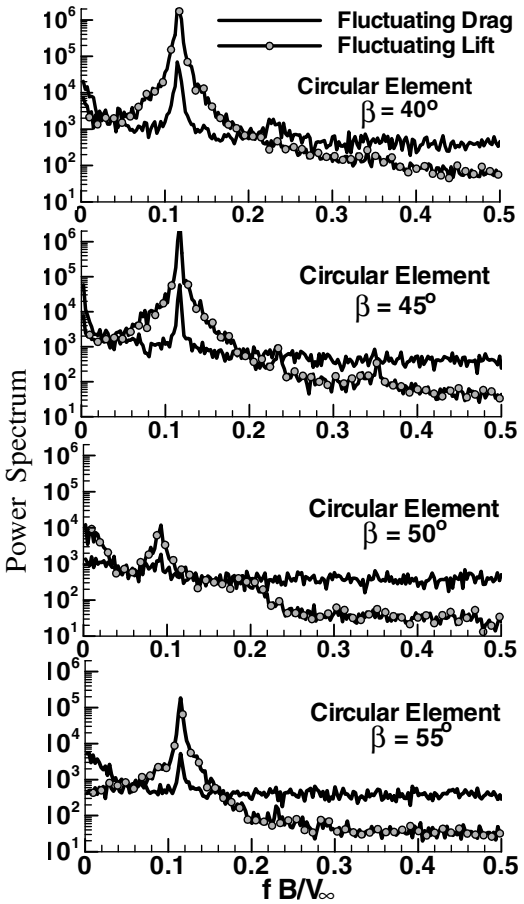

b)

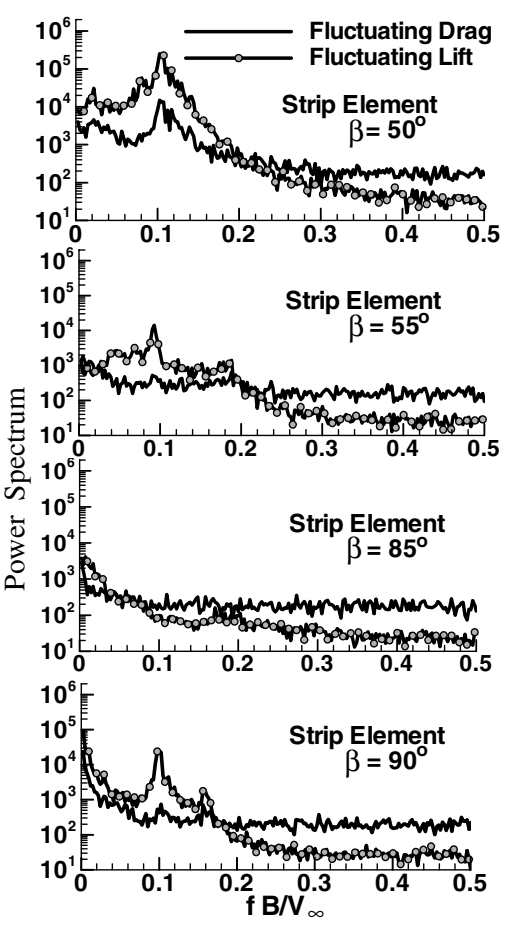

c)

Fig. 18 Angle $\alpha=\beta$ effect of the three elements of $b / B=0.24$ on power spectra of fluctuating lift and drag coefficients of the cylinder, $\lambda / B=1.31$, $R e=5.6 \times 10^{4}$, and the blockage ratio is $8.27 \%$.

is removed in the effective range of angle $\alpha=\beta=45-70 \mathrm{deg}$. The sharp peak reappears in the range $\alpha=\beta=75-90 \mathrm{deg}$, in which monoside vortex shedding occurs; however, the peak value is lower than that without element.

The strip element of $b / B=0.24$ has a similar $\alpha=\beta$ effect on the spectra of fluctuating lift (Fig. 18c), but the range of angle $\alpha=\beta$ for elimination of the sharp peak is 55-85 deg. The sharp peak appears again in the vicinity of $\alpha=\beta=90 \mathrm{deg}$.

The suppression effect of the circular element of $b / B=0.24$ is poor. As shown in Fig. 18b, the sharp peak in the spectrum of fluctuating lift can only be markedly reduced in a very narrow range in the vicinity of $\alpha=\beta=50 \mathrm{deg}$. Similar changes happen to the spectrum of fluctuating drag with $\alpha=\beta$, but the peak values of the spectra with and without element are much smaller that those of fluctuating lift.

The effects of angle $\alpha=\beta$ of the three elements of $b / B=0.24$ on the rms value of fluctuating lift coefficients $C_{l}^{\prime}$ are shown in Fig. 19a.
$C_{l}^{\prime}$ is reduced by $83-93 \%$ when the square element is applied and set in the range of $\alpha=\beta=40-70 \mathrm{deg}$. It is reduced by $91 \%$ when the strip element is used and set in $\alpha=\beta=55-85 \mathrm{deg}$. However, the reduction rate of $C_{l}^{\prime}$ is much smaller at most of the $\alpha=\beta$ angles when the circular element is applied.

$C_{d}^{\prime}$ can be reduced at all $\alpha=\beta$ angles by the square or strip element (Fig. 19b). It can be slightly reduced at $\alpha=\beta=0-20$ and 50-90 deg, but increased at $\alpha=\beta=25-45 \mathrm{deg}$ if the circular element is used.

Abrupt changes in $C_{l}^{\prime}$ and $C_{d}^{\prime}$ are induced by the circular element at $\alpha=\beta \approx 50 \mathrm{deg}$, but no abrupt changes are induced by the other two elements.

\section{G. Effects on Mean Forces}

The effect of angle $\alpha=\beta$ of the three elements of $b / B=0.24$ on the mean drag and lift coefficients are shown in Figs. 20a and 20b, respectively. The mean drag $C_{D}$ is reduced by $21-25 \%$ when the
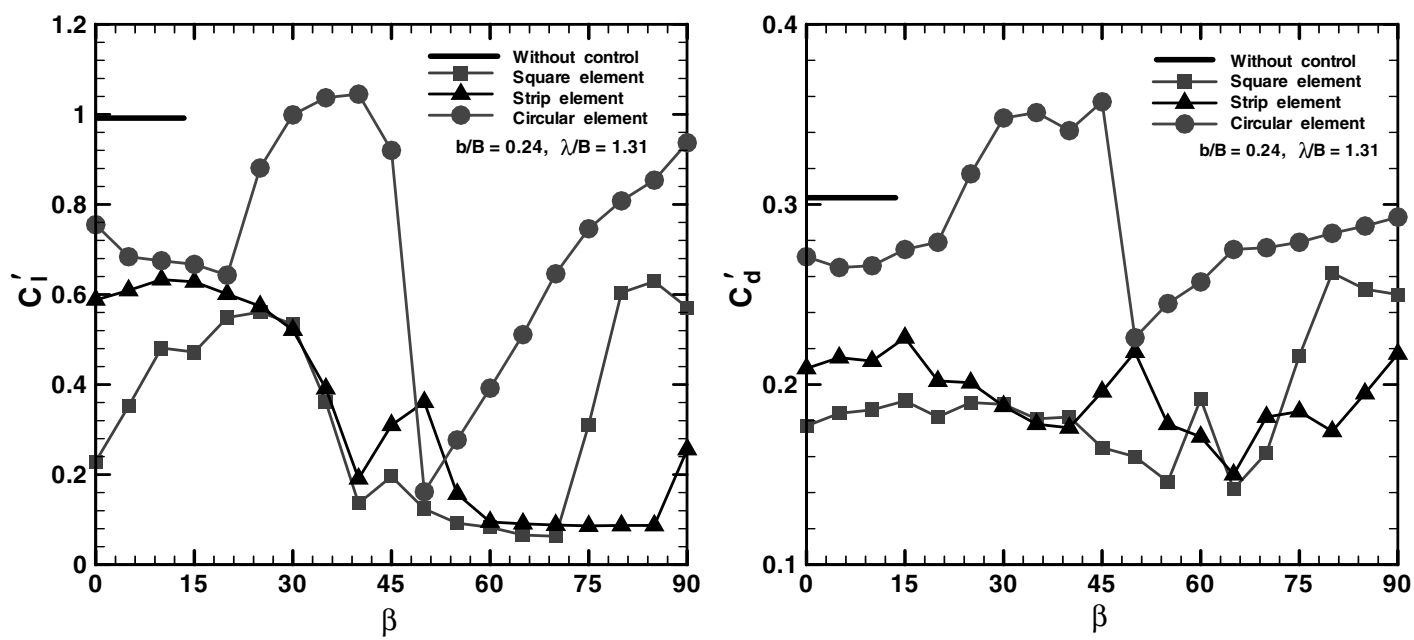

a) Effects on fluctuating lift coefficient

b) Effects on fluctuating drag coefficient

Fig. 19 Angle $\alpha=\beta$ effect of the three elements of $b / B=0.24$ on fluctuating lift and drag coefficients $C_{l}^{\prime}$ and $C_{d}^{\prime}, \lambda / B=1.31, R e=5.6 \times 10^{4}$, and the blockage ratio is $8.27 \%$. 


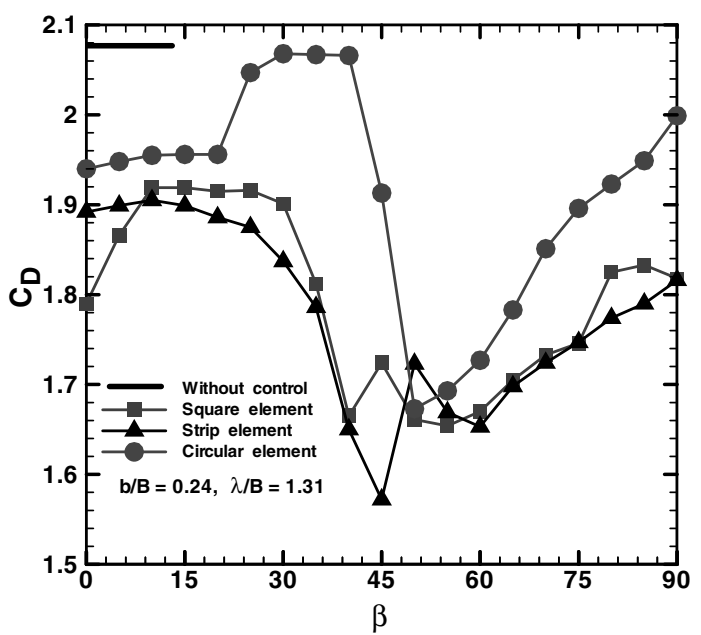

a) Mean drag coefficient

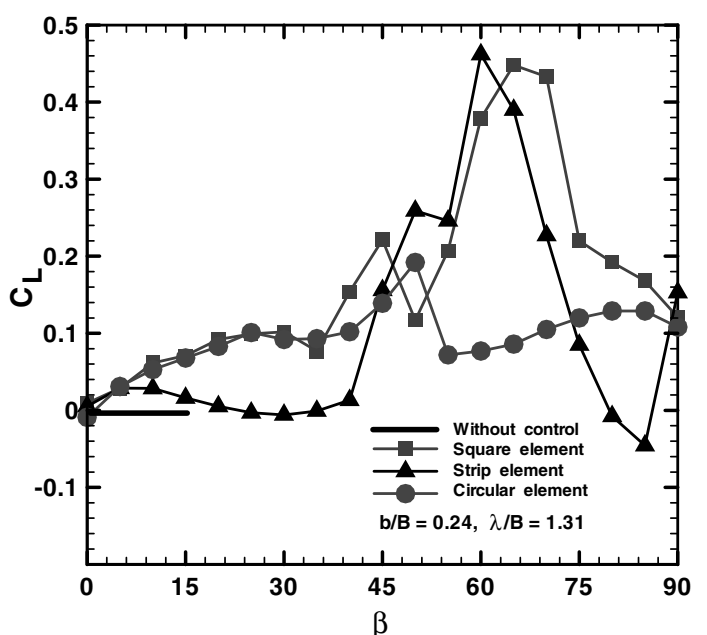

b) Mean lift coefficient

Fig. $20 \alpha=\beta$ angle effects of the three elements of $b / B=0.24$ on mean drag and lift coefficients, $\lambda / B=1.31, R e=5.6 \times 10^{4}$, and the blockage ratio is 8.27\%.

square or the strip element is applied in the range of $\alpha=\beta=40-70 \mathrm{deg}$. It is reduced by $17-20 \%$ when the circular element is set in the range of $\alpha=\beta=50-60 \mathrm{deg}$. The mean lift coefficient $C_{L}$ is increased to 0.45 by the square or strip element at $\alpha=\beta=50-75 \mathrm{deg}$. $C_{L}$ is small at other $\alpha=\beta$ angles. The maximum value of $C_{L}$ is only 0.2 when the circular element is applied.

All of the preceding results indicate that the strip and square elements are much more effective than the circular element in suppression of vortex shedding, elimination of fluctuating forces, reduction of mean drag, and increase of mean lift.

\section{Discussions}

The mechanism of vortex-shedding suppression by a circular element was discussed in Strykowski and Sreenivasan [3] based on Gerrard's [7] model of vortex generation. The model demonstrates the main role of interaction between the separated shear layers in vortex formation and shedding-frequency selection. The function of the element is said to diffuse the vorticity in one shear layer [3]. The circulation on the diffused side is then reduced below a threshold, so that the mutual attraction between the opposing shear layers is too weak to form the vortex roll-up on both sides. It is argued by Gerrard [7] that a stronger attraction of the shear layers produces a higher frequency of vortex shedding, and a weaker attraction results in a lower frequency. However, the phenomenon of monoside vortex shedding, as well as its shedding frequency (shown in Figs. 2, $\underline{3}$, and 7) runs counter to Gerrard's model and Strykowski and Sreenivasan's [3] deduction.

The properties of bluff-body wakes have been investigated by introducing the concepts of global instability and local absolute and convective instability [14]. Stability analyses $[15,16]$ have indicated that there is a region in the near wake of a bluff body in which the velocity profiles are absolutely unstable. Relations of the circularelement suppression to local absolute instability, temporally growing global modes, and vortex shedding have all been explored by Strykowski and Sreenivasan [3], but have not yet been firmly established. Later advances in stability theory of weakly nonparallel flows $[17,18]$ have shown that a sufficiently large region of absolute instability is responsible for global instability and vortex generation. Test results show that the velocity profiles in the near wake are altered locally by the presence of an element [6]. The local modifications of the profiles may induce changes in their stability nature. We may deduce that if the element is set in the effective zone, the absoluteinstability region in the wake can be eliminated or reduced to a sufficiently small level so that large-scale vortex cannot generate in the near wake. The velocity profiles in the near wake are altered more by an element of larger bluffness and altered less by an element of smaller bluffness. This may be the reason behind the differences in size and location of the effective zone between different elements, but it has to be proved by further study.

\section{Conclusions}

The control method of using a small circular element proposed by Strykowski and Sreenivasan has a poor effect on suppression of vortex shedding at Reynolds numbers higher than the order of $10^{2}$. A small square or a narrow strip element was introduced in the present study to improve the suppression of vortex shedding from a square cylinder at test Reynolds numbers in the range of $1.12 \times 10^{4}-1.02 \times 10^{5}$. Experimental results show that vortex shedding from both sides of the cylinder can be suppressed if the element size is greater than a critical value, the angle of attack of the element is in a certain range, and the position of the element is within an effective zone. The bluffness of the element plays an important role in the control. The larger the bluffness, the larger the effective zone of element position. The effective zone moves upstream and inward with the increase of blockage ratio from 4.13 to $12.40 \%$. The effective zone slightly enlarges with the increase of Reynolds number in the range tested. A phenomenon of monoside vortex shedding is observed that cannot be readily explained by Gerrard's [7] model of vortex generation. The control mechanism is briefly discussed from a wake-stability point of view.

\section{Acknowledgments}

This study was financially supported by the Natural Science Foundation of China, grant nos. 10172087 and 10472124. The authors wish to thank Jianming Wang, Senior Engineer Bin Liang, Kai Chen, Engineer Fengrong Zhu, and Ya-Li Wang for their help in preparing the experiment.

\section{References}

[1] Zdravkovich, M. M., "Review and Classification of Various Aerodynamic and Hydrodynamic Means for Suppressing Vortex Shedding," Journal of Wind Engineering and Industrial Aerodynamics, Vol. 7, No. 2, 1981, pp. 145-189. doi:10.1016/0167-6105(81)90036-2

[2] Gad-el-Hak, M., Flow Control: Passive, Active, and Reactive Flow Management, Cambridge Univ. Press, New York, 2000, pp. 150-188.

[3] Strykowski, P. J., and Sreenivansan, K. R., "On the Formation and Suppression of Vortex Shedding at Low Reynolds Numbers," Journal of Fluid Mechanics, Vol. 218, Sept. 1990, pp. 71-107. doi:10.1017/S0022112090000933

[4] Shao, C.-P., and Wang, J.-M., "Control of Vortex Shedding at Relatively High Reynolds Numbers," Chinese Journal of Theoretical and Applied Mechanics, Vol. 38, No. 2, 2006, pp. 153-161 (in 
Chinese).

[5] Shao, C.-P., Wang, J.-M., and Wei, Q.-D., "Visualization Study on Suppression of Vortex Shedding from a Cylinder," Journal of Visualization, Vol. 10, No. 1, 2007, pp. 57-64. doi:10.1007/s00791-006-0048-3

[6] Shao, C.-P., and Wang, J.-M., "Control of Mean and Fluctuating Forces on a Circular Cylinder at High Reynolds Numbers," Acta Mechanica Sinica, Vol. 23, No. 2, 2007, pp. 133-143. doi:10.1007/s10409-007-0055-8

[7] Gerrard, J. H., "The Mechanics of the Formation Region of Vortices Behind Bluff Bodies," Journal of Fluid Mechanics, Vol. 25, Pt. 2, 1966, pp. 401-413. doi:10.1017/S0022112066001721

[8] Unal, M. F., and Rockwell, D., "On Vortex Formation from a Cylinder, Part 2: Control by Splitter-Plate Interference," Journal of Fluid Mechanics, Vol. 190, May 1988, pp. 513-529. doi:10.1017/S0022112088001430

[9] Igarashi, T., "Characteristics of the Flow Around a Square Prism," Bulletin of the JSME, Vol. 27, No. 231, 1984, pp. 1858-1865.

[10] Munshi, S. R., Modi, V. J., and Yokomizo, T., "Fluid Dynamics of Flat Plates and Rectangular Prisms in the Presence of Moving Surface Boundary Layer Control," Journal of Wind Engineering and Industrial Aerodynamics, Vol. 79, Nos. 1-2, 1999, pp. 37-60. doi:10.1016/S0167-6105(97)00294-8

[11] Roshko, A., "On the Wake and Drag of Bluff Bodies," Journal of the Aeronautical Sciences, Vol. 22, No. 2, 1955, pp. 124-132.

[12] Chen, J. M., and Fang, Y. C., "Strouhal Numbers of Inclined Flat Plates," Journal of Wind Engineering and Industrial Aerodynamics, Vol. 61, Nos. 2-3, 1996, pp. 99-112.
doi:10.1016/0167-6105(96)00044-X

[13] Fage, A., and Johansen, F. C., "On the Flow of Air Behind an Inclined Flat Plate of Infinite Span," Proceedings of the Royal Society of London A, Vol. 116, No. A773, 1927, pp. 170-197. doi:10.1098/rspa.1927.0130

[14] Huerre, P., and Monkewitz, P. A., "Local and Global Instabilities in Spatially Developing Flows," Annual Review of Fluid Mechanics, Vol. 22, 1990, pp. 473-537. doi:10.1146/annurev.fl.22.010190.002353

[15] Koch, W., "Local Instability Characteristics and Frequency Determination of Self-Excited Wake Flows," Journal of Sound and Vibration, Vol. 99, No. 1, 1985, pp. 53-83. doi:10.1016/0022-460X(85)90445-6

[16] Triantafyllou, G. S., Triantafyllou, M. S., and Chryssotomidis, C., "On the Formation of Vortex Streets Behind Stationary Cylinders," Journal of Fluid Mechanics, Vol. 170, Sept. 1986, pp. 461-477. doi:10.1017/S0022112086000976

[17] Monkewitz, P. A., Huerre, P., and Chomaz, J. M., "Global Linear Stability Analysis of Weakly Nonparallel Shear Flows," Journal of Fluid Mechanics, Vol. 251, June 1993, pp. 1-20. doi:10.1017/S0022112093003313

[18] Chomaz, J. M., "Global Instabilities in Spatially Developing Flows: Nonnormality and Nonlinearity," Annual Review of Fluid Mechanics, Vol. 37, 2005, pp. 357-392. doi:10.1146/annurev.fluid.37.061903.175810

N. Chokani Associate Editor 